Why does yeast ferment? A flux balance analysis study

Simeonidis, Evangelos and Murabito, Ettore and Smallbone, Kieran and Westerhoff, Hans V.

2010

MIMS EPrint: 2010.85

Manchester Institute for Mathematical Sciences

School of Mathematics

The University of Manchester

\footnotetext{
Reports available from: http://eprints.maths.manchester.ac.uk/

And by contacting: The MIMS Secretary

School of Mathematics

The University of Manchester

Manchester, M13 9PL, UK
} 


\title{
Why does yeast ferment? A flux balance analysis study
}

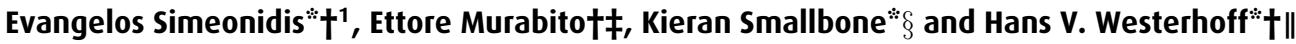

"Manchester Centre for Integrative Systems Biology, Manchester Interdisciplinary Biocentre, 131 Princess Street, Manchester M1 7DN, U.K., †School of Chemical Engineering and Analytical Science, University of Manchester, Oxford Road, Manchester M13 9PL, U.K., †Doctoral Training Centre Integrative Systems Biology from Molecules to Life, Manchester Interdisciplinary Biocentre, University of Manchester, 131 Princess Street, Manchester M1 7DN, U.K., $\S S$ chool of Mathematics, University of Manchester, Oxford Road, Manchester M13 9PL, U.K., and \#Department of Molecular Cell Physiology, Vrije Universiteit, de Boelelaan 1085, 1081 HV Amsterdam, The Netherlands

\begin{abstract}
Advances in biological techniques have led to the availability of genome-scale metabolic reconstructions for yeast. The size and complexity of such networks impose limits on what types of analyses one can perform. Constraint-based modelling overcomes some of these restrictions by using physicochemical constraints to describe the potential behaviour of an organism. FBA (flux balance analysis) highlights flux patterns through a network that serves to achieve a particular objective and requires a minimal amount of data to make quantitative inferences about network behaviour. Even though FBA is a powerful tool for system predictions, its general formulation sometimes results in unrealistic flux patterns. A typical example is fermentation in yeast: ethanol is produced during aerobic growth in excess glucose, but this pattern is not present in a typical FBA solution. In the present paper, we examine the issue of yeast fermentation against respiration during growth. We have studied a number of hypotheses from the modelling perspective, and novel formulations of the FBA approach have been tested. By making the observation that more respiration requires the synthesis of more mitochondria, an energy cost related to mitochondrial synthesis is added to the FBA formulation. Results, although still approximate, are closer to experimental observations than earlier FBA analyses, at least on the issue of fermentation.
\end{abstract}

\section{Introduction}

Systems biology studies the function and behaviour of biological systems and the interactions among the components of these systems; for example, metabolic networks and the interactions of the metabolites and enzymes that constitute them. Such systems exhibit highly non-linear characteristics, leading to very complex system dynamics [1-3]. In addition, advances in high-throughput experimentation and bioinformatics have led to an explosion of '-omics' data availability. The development of rigorous quantitative methods is fast becoming a necessity in order to build comprehensive theoretical models for interpretation, organization and integration of these data to guide experimental design and understanding.

Whereas the first stoichiometric models for baker's yeast (Saccharomyces cerevisiae) were limited to the central metabolic pathways, later genome-scale efforts [4-7] were much more comprehensive. The size and complexity of such networks often poses a serious restriction on what type of analyses we are able to perform on metabolism. Constraintbased modelling $[8,9]$ overcomes such limitations by using the stoichiometry of the metabolic network and physicochemical

Key words: energy cost for mitochondrial synthesis, fermentation, flux balance analysis (FBA) overflow metabolism, respiration, Saccharomyces cerevisiae.

Abbreviations used: ATPC, ATP consumption; DW, dry weight; FBA, flux balance analysis; TCA tricarboxylic acid.

${ }^{1}$ To whom correspondence should be addressed (email v.simeonidis@manchester.ac.uk). constraints such as mass balance, energy balance and flux considerations to describe the potential behaviour of an organism. A number of stoichiometric techniques have been proposed, including FBA (flux balance analysis), extreme pathway analysis or elementary mode analysis.

In particular, FBA [10] highlights the optimal path through the network in order to achieve a particular objective, such as the maximization of biomass. The key benefit lies in the minimal amount of biological data required to make quantitative inferences. Although FBA is a very powerful tool for system behaviour predictions (for example, in flux optimization of engineered processes [11,12]), this comes at a price. FBA is concerned only with fluxes through the system and does not make predictions about metabolic concentrations. Also, there is degeneracy in its solutions, leading to a large number of alternative flux patterns with the same optimal value. Finally, the general FBA formulation sometimes results in unrealistic flux patterns, a typical example of which is alcoholic fermentation in yeast; even though it is well known that yeast normally produces ethanol during growth, this flux pattern is not present in a typical FBA solution.

Elsewhere, we have examined the problems of lack of information for metabolic concentrations $[13,14]$ and solution degeneracy $[15,16]$ in FBA. In the present paper, we examine the issue of unrealistic solutions, and specifically fermentation against respiration in baker's yeast. We have 
studied alternative conditions and optimization pressures for the model in order to observe the expected fermentation. We propose three improvements for the FBA formulation, which allow us to obtain the observed fermentative behaviour in the model. Our hypotheses are tested and conclusions are drawn.

\section{Flux balance analysis}

Mathematically, FBA is framed as an LP (linear programming) problem, Formulation I:

$$
\text { Maximize } Z=v_{\text {growth }}
$$

subject to:

$$
\begin{aligned}
& \sum_{j} N_{i j} \cdot v_{j}=0 \quad \forall_{i} \\
& v_{j}^{\min } \leqslant v_{j} \leqslant v_{j}^{\max } \quad \forall_{i}
\end{aligned}
$$

where $i$ is the set of metabolites, $j$ is the set of reactions in the network, $N_{i j}$ is the stoichiometric matrix, and $v_{\mathrm{j}}$ is the flux of reaction $j$. The objective function (1) is maximized over all possible steady-state fluxes satisfying certain constraints (2). In genome-scale metabolic models, a biomass producing reaction is defined and used as the objective function. Upper and lower bounds are placed on the individual fluxes (3). For irreversible reactions, $v^{\min }=0$. Specific upper bounds, based on enzyme capacity measurements, may be imposed on reactions; in the absence of any information, these rates can be generally assumed unconstrained i.e. $v^{\max }=\infty$ and $v^{\min }=-\infty$ for reversible reactions. To avoid unbounded solutions, i.e. $Z$ reaching $\infty$, one rate (the input flux) needs to be fixed to a specific value, and all fluxes should be viewed as relative to the input flux. Here, we fix the influx of glucose, but not always at the same level. For the simulations in the present paper, we used the iND750 genome-scale model of yeast metabolism [5].

\section{Hypoxic conditions}

For yeast, the ability to ferment sugars is a necessity for growth under anaerobic conditions. When a hexose is imported into the cell, it is broken down by glycolysis into two molecules of pyruvate. During glycolysis, there is a net production of two molecules of ATP and two molecules of $\mathrm{NADH}$. Under aerobic conditions, NAD is regenerated by transfer of the electrons of NADH to Complex I (NADH:Q oxidoreductase, proton-translocating) of the respiratory chain, from where the electrons continue to flow to molecular oxygen. In $S$. cerevisiae, the process of respiration results in the reduction of molecular oxygen to water [17] and the generation of a proton gradient along the mitochondrial membrane. This gradient drives ATP synthesis [18]. In the absence of oxygen, the enzymes pyruvate decarboxylase and alcohol dehydrogenase convert pyruvate into ethanol and $\mathrm{CO}_{2}$ to reoxidize the two molecules of
Figure 1 Activation of fermentation under hypoxic conditions

(a) Fluxes through the TCA cycle and the fermentative pathway against glucose uptake under hypoxic conditions. (b) Flux of the TCA cycle and growth rate against glucose uptake under hypoxic conditions. GLC, glucose.
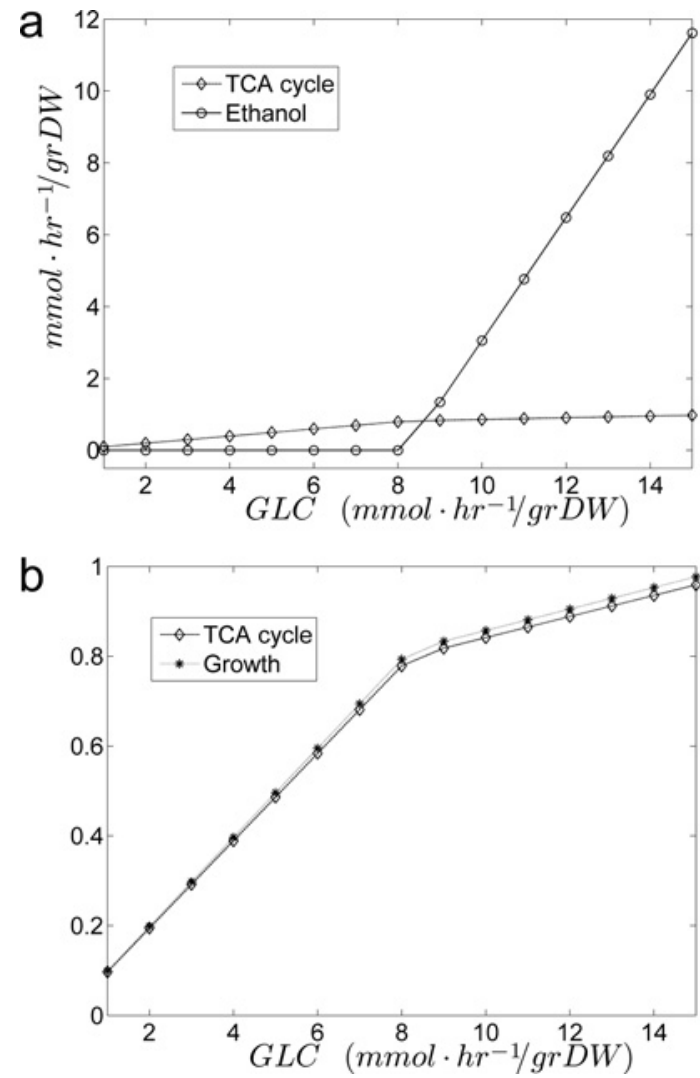

$\mathrm{NADH}$, which were produced in glycolysis [19]. During the latter process, known as alcoholic fermentation, two ATP molecules are formed from one molecule of glucose. Under anaerobic conditions (i.e. when we forbid oxygen uptake in the model), the solution of Formulation I showed a switch from respiration to fermentation, and ethanol was produced. This was to be expected, as the complete lack of oxygen does not allow the respiratory reactions to progress, so an alternative for the reoxidation of NADH was found by the model.

We then tested the hypothesis that fermentation could also be a response to relative oxygen limitation, i.e. when oxygen levels decline more rapidly than glucose levels (or glucose availability increases more rapidly than oxygen). To emulate these conditions in a FBA problem, oxygen transport (unbounded in the original model) was given an upper bound [20 $\mathrm{mmol} \cdot \mathrm{h}^{-1} / \mathrm{g}$ of DW (dry weight)]. Formulation I was solved several times, each time increasing the uptake flux of glucose. We observed that when the rate of glucose uptake increased over a certain threshold $\left(\sim 8 \mathrm{mmol} \cdot \mathrm{h}^{-1} / \mathrm{g}\right.$ of DW), production of ethanol was activated and subsequently increased linearly with the rate at which glucose entered the system (Figure 1a). 
Figure 1(b) shows that the biomass production was dependent on the flux through the TCA (tricarboxylic acid) cycle even when the fermentative pathway had been activated, indicating that there is no switch from the respiratory to the fermentative pathway as the uptake of glucose increases. Rather, our FBA solution predicts that fermentation is activated on top of respiration, because of the limited availability of oxygen, which becomes insufficient to process all of the glucose through the TCA cycle. These results are reminiscent of overflow metabolism [20], a phenomenon in which $S$. cerevisiae is subjected to an increased glycolytic flux and metabolism shifts from pure respiration to a mix of respiratory and fermentative behaviour, even under aerobic conditions.

\section{Minimization of the number of active reactions}

We next tested the hypothesis that, instead of optimal growth, the 'driving force' for metabolism is resource preservation, i.e. we made the sensible assumption that the cell will minimize the number of active reactions (and therefore the enzymes that need to be synthesized) necessary to produce the required biomass [21]. Formulation I was solved to identify a value for biomass production $(Z *)$. This value was then fixed and Formulation II was devised:

$$
\text { Minimize } \sum_{j} y_{j}
$$

subject to:

$$
\begin{aligned}
& v_{\text {growth }}=Z^{*} \\
& \sum_{j} N_{i j} \cdot v_{j}=0 \quad \forall_{i} \\
& y_{j} \cdot v_{j}^{\min } \leqslant v_{j} \leqslant y_{j} \cdot v_{j}^{\max } \forall_{j}
\end{aligned}
$$

The binary (decision) variable $y_{j}$ is equal to 1 when reaction $j$ is active and equal to 0 otherwise. The new objective (4) represents the total number of active reactions in the network, where an 'active' reaction is defined as one with a non-zero flux. Constraint (7) is a modified form of constraint (3); the implication of binary variable $y_{j}$ is that the flux of reaction $j$ can only vary between $v^{\min }$ and $v^{\max }$ when $y_{j}$ is not equal to 0 . Formulation II, which is a MILP (mixed integer linear programming) problem, was solved for increasing values of glucose uptake.

The results are presented in Figure 2. When glucose uptake was below a certain threshold, the system used the respiratory pathway to produce the required amount of ATP in order to maintain biomass production equal to $Z *$. When glucose uptake was sufficiently high so that the ATP produced by glycolysis became adequate to guarantee the required growth rate, the system switched from respiration to fermentation
Figure 2 Switch from respiration to fermentation under resource preservation

(a) Normalized fluxes through the TCA cycle and the fermentative pathway against glucose uptake while minimizing the number of active reactions. (b) Normalized flux of the TCA cycle and normalized growth rate against glucose uptake while minimizing the number of active reactions. GLC, glucose.
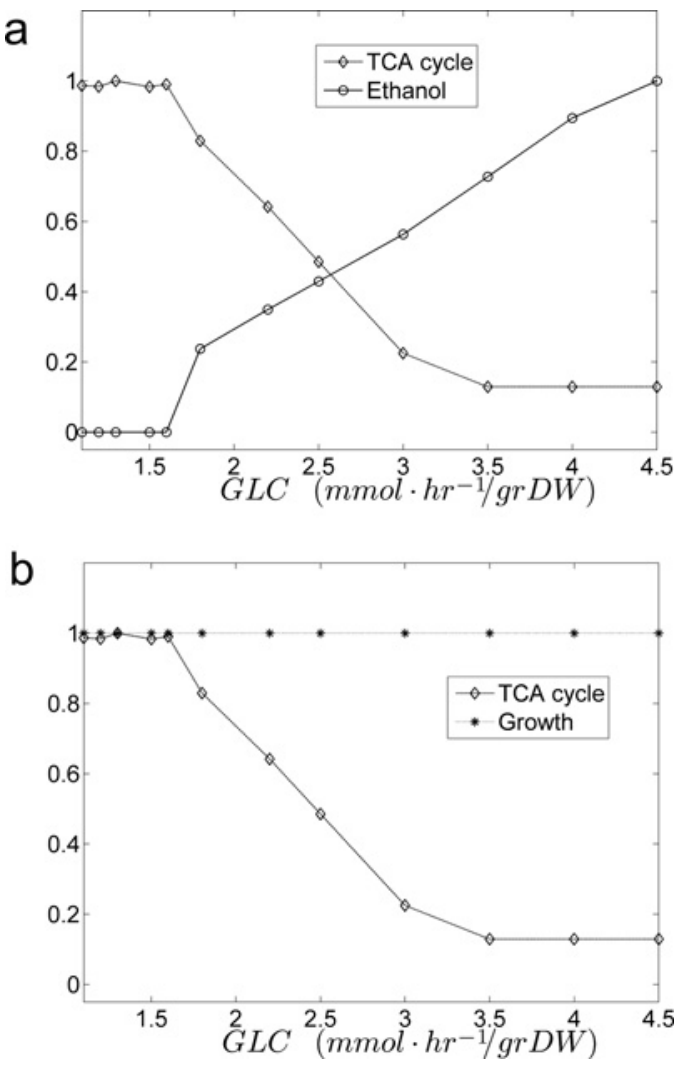

in order to minimize the number of active reactions and conserve resources. The hypothesis underlying these results (i.e. the tendency to minimize the resources needed to guarantee a certain biomass production rate) may provide an explanation for the Crabtree effect [22], an experimentally observed behaviour whereby $S$. cerevisiae produces ethanol aerobically in the presence of high external glucose concentrations rather than producing biomass through the TCA cycle. However, one should remain aware that, during our optimization for the minimum number of reaction steps, we assumed the growth rate (and yield) to be constant, which may or may not correspond to evolutionary reality.

\section{Energy costs related to mitochondrial synthesis}

Respiring is a more efficient method of producing ATP molecules than fermentation. Aerobic respiration breaks down pyruvate produced through glycolysis and requires that pyruvate enters the mitochondrion in order to be fully oxidized by the TCA cycle. The product of this process is Gibbs free energy in the form of ATP. Nevertheless, 
the choice for more respiration necessitates the synthesis of more mitochondria, and, in addition, a number of enzymes involved in the TCA cycle, respiratory chain and in ATP synthesis. The energy cost of these processes is not taken into account in the traditional FBA approach. To consider this aspect, we examined the hypothesis that the necessary mitochondria synthesis adds an 'ATP cost' to the system. An ATPC (ATP consumption) reaction was included in the model, which was used to account for any ATP losses due to cellular operations:

$$
\text { ATPC reaction: } \mathrm{ATP}+\mathrm{H}_{2} \mathrm{O} \rightarrow \mathrm{ADP}+\mathrm{H}^{+}+\mathrm{P}_{\mathrm{i}}
$$

The value of the flux of this reaction is typically given a lower bound of $1 \mathrm{mmol} \cdot \mathrm{h} / \mathrm{g}$ of DW [4].

$$
1 \leqslant v_{\text {ATPC }} \leqslant v_{\text {ATPC }}^{\max }
$$

Constraint (8) is the specialized form of constraint (3) for reaction ATPC. In order to consider an energy cost for the synthesis of mitochondria, we altered constraint (8) as follows:

$$
1+c_{\mathrm{Mit}} \cdot \sum_{j \in \mathrm{Mit}} v_{j} \leqslant v_{\mathrm{ATPC}} \leqslant v_{\mathrm{ATPC}}^{\max }
$$

where $c_{\text {Mit }}$ is an energy cost associated with mitochondrial use, and $\sum_{j \in \text { Mit }} v_{j}$ represents the mitochondrial flux (the total flux of all active reactions in the mitochondria). The transformation presented above penalizes the use of mitochondrial flux by increasing the related consumption of ATP. Formulation I was solved with the addition of constraint (9). A sensitivity analysis was performed to identify appropriate values of $c_{\text {Mit }}$, the results of which are presented in Figure 3.

Figure 3 shows the existence of a bifurcation point for $c_{\text {Mit }} \approx 0.14$. Below this value, the model used the respiratory pathway, as this allowed for the production of more ATP. Above this value, there was a sharp switch to the fermentative pathway, as the associated energy cost made fermentation the optimal choice.

\section{Conclusions}

In studying the problem of FBA formulations predicting respiration instead of fermentation during growth in yeast, we have outlined three hypotheses in order to make our model predict the expected activity of the fermentative pathway. First, we explored the behaviour of the model under conditions of oxygen limitation. We concluded that such conditions do indeed activate the fermentative pathway, but as an additional route for the uptake of glucose and not as an alternative to respiration, perhaps an instance of overflow metabolism. However, the Crabtree effect is also observed in yeast cells with ample oxygenation. Therefore the hypothesis that the cell would minimize the number of active reactions in attempting to optimize resource management was tested. Again, our model predicted ethanol production at higher levels of glucose uptake, as the fermentative pathway
Figure 3 Sensitivity analysis of fermentative response to mitochondrial cost

(a) Normalized fluxes through the TCA cycle and the fermentative pathway against mitochondrial cost. (b) Normalized flux of the TCA cycle and normalized growth rate against mitochondrial cost.
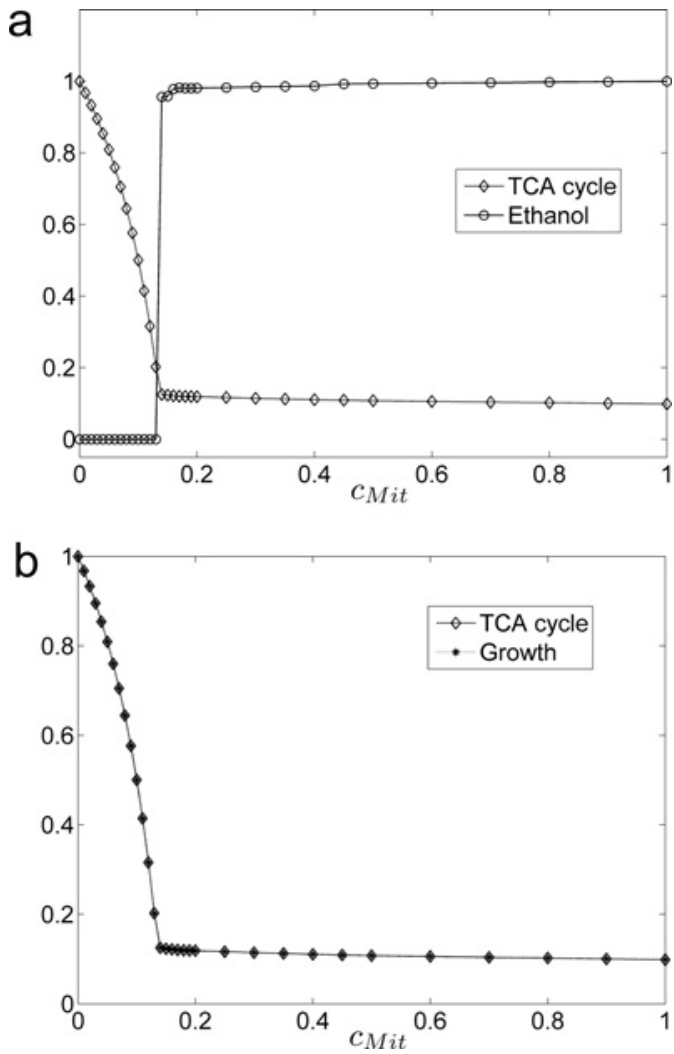

requires a considerably smaller number of enzymes and pathways, suggesting a possible explanation for the Crabtree effect. However, it is unclear why there should be an optimum imposed on the number of enzymes, if it were not for the free energy required to synthesize them [23], or for competition for the synthesis of other proteins at the level of the ribosome [24,25]. Finally, we tested the hypothesis that more respiration requires the synthesis of more mitochondria, or numerous TCA cycle enzymes, and a free energy cost tied to the total mitochondrial flux was added to the formulation. The simulation predicted that the fermentative pathway is now dominant, provided that the assumed energy cost for the synthesis of mitochondria (or at the very least, for the synthesis of TCA cycle enzymes involved in the respiratory chain and in ATP synthesis) is above a specific value. The results of the simulations can be used to design experiments that could help to decide which of the three model behaviours described is closer to experimental observation. Alternatively, it could be the case that such prediction failures are caused by FBA's unicellular analysis, missing important cell-cell interactions. For example, the production of ethanol could be a waste product toxic to other cells, an evolutionary mechanism favouring output over efficiency in the competition for nutrients. 
Current work focuses on determining a value for the ATPrelated cost for the synthesis of mitochondria, $c_{\mathrm{Mit}}$, based on either experimental or literature evidence. We also want to extend the method to take into account the dependence of mitochondrial synthesis on the total metabolic flux, a consideration that would make the FBA formulation an NLP (non-linear programming) problem. Our results, although still approximate and based on many of the same assumptions as traditional FBA simulations, are closer to experimental observations than those classical simulations were [26], at least on the issue of yeast fermentation. Such crucial improvements are a necessary stepping stone for the creation of a realistic genome-scale model, and may thus be considered part of the deductive-inductive 'cycle of knowledge' [27] crucial for systems biology: by using an approximative model of the system, one can guide experimental design and can subsequently update the model as specific knowledge becomes available from experimental results.

\section{Acknowledgements}

We thank our colleagues in the Manchester Centre for Integrative systems Biology.

\section{Funding}

This work is funded by the Biotechnology and Biological Sciences Research Council and Engineering and Physical Sciences Research Council [grant numbers BBD0190791, BBC0082191, BBF0035281, BBF0035521, BBF0035361, BBG5302251 and SysMO P 49], European Union Framework Programme 7 (BioSim, NucSys, EC-MOAN) and other funders (http://www.systembiology.net/support/).

\section{References}

1 Lazebnik, Y. (2002) Can a biologist fix a radio? Or, what I learned while studying apoptosis. Cancer Cell 2, 179-182

2 Mendes, P. and Kell, D. (1998) Non-linear optimization of biochemical pathways: applications to metabolic engineering and parameter estimation. Bioinformatics 14, 869-883

3 Szallasi, Z., Stelling, J. and Periwal, V. (2006) System Modeling in Cellular Biology: from Concepts to Nuts and Bolts, MIT Press, Boston, MA

4 Forster, J., Famili, I., Fu, P., Palsson, B.Ø. and Nielsen, J. (2003) Genome-scale reconstruction of the Soccharomyces cerevisioe metabolic network. Genome Res. 13, 244-253

5 Duarte, N.C., Herrgard, M.J. and Palsson, B.Ø. (2004) Reconstruction and validation of Saccharomyces cerevisioe iND750, a fully compartmentalized genome-scale metabolic model. Genome Res. 14, 1298-1309

6 Nookaew, I., Jewett, M.C., Meechai, A., Thammarongtham, C., Laoteng, K., Cheevadhanarak, S., Nielsen, J. and Bhumiratana, S. (2008) The genome-scale metabolic model ilN800 of Sacchoromyces cerevisioe and its validation: a scaffold to query lipid metabolism. BMC Syst. Biol. 2, 71
7 Herrgard, M.J., Swainston, N., Dobson, P., Dunn, W.B., Arga, K.Y., Arvas, M., Bluthgen, N., Borger, S., Costenoble, R., Heinemann, M. et al. (2008) A consensus yeast metabolic network reconstruction obtained from a community approach to systems biology. Nat. Biotechnol. 26, $1155-1160$

8 Covert, M.W., Famili, I. and Palsson, B.Ø. (2003) Identifying constraints that govern cell behavior: a key to converting conceptual to computational models in biology? Biotechnol. Bioeng. 84, 763-772

9 Price, N.D., Reed, J.L. and Palsson, B.Ø. (2004) Genome-scale models of microbial cells: evaluating the consequences of constraints. Nat. Rev. Microbiol. 2, 886-897

10 Kauffman, K.J., Prakash, P. and Edwards, J.S. (2003) Advances in flux balance analysis. Curr. Opin. Biotechnol. 14, 491-496

11 Edwards, J.S. and Palsson, B.Ø. (2000) The Escherichio coli MG1655 in silico metabolic genotype: its definition, characteristics, and capabilities Proc. Natl. Acad. Sci. U.S.A. 97, 5528-5533

12 Schilling, C.H. and Palsson, B.Ø. (2000) Assessment of the metabolic capabilities of Hoemophilus influenzoe Rd through a genome-scale pathway analysis. J. Theor. Biol. 203, 249-283

13 Smallbone, K., Simeonidis, E., Broomhead, D.S. and Kell, D.B. (2007) Something from nothing: bridging the gap between constraint-based and kinetic modelling. FEBS J. 274, 5576-5585

14 Smallbone, K., Simeonidis, E., Swainston, N. and Mendes, P. (2010) Towards a genome-scale kinetic model of cellular metabolism. BMC Syst. Biol. 4, 6

15 Murabito, E., Simeonidis, E., Smallbone, K. and Swinton, J. (2009) Capturing the essence of a metabolic network: a flux balance analysis approach. J. Theor. Biol. 260, 445-452

16 Smallbone, K. and Simeonidis, E. (2009) Flux balance analysis: a geometric perspective. J. Theor. Biol. 258, 311-315

17 Yagi, T., Seo, B.B., Di Bernardo, S., Nakamuru-Ogiso, E., Kao, M.C. and Matsuno-Yagi, A. (2001) NADH dehydrogenases: from basic science to biomedicine. J. Bioenerg. Biomembr. 33, 233-242

18 Mitchell, P. (1966) Chemiosmotic coupling in oxidative and photosynthetic phosphorylation. Biol. Rev. Cambridge Philos. Soc. 41, 445-502

19 Barnett, J.A. (2003) A history of research on yeasts 5: the fermentation pathway. Yeast 20, 509-543

20 Vemuri, G.N., Eiteman, M.A., McEwen, J.E., Olsson, L. and Nielsen, J. (2007) Increasing NADH oxidation reduces overflow metabolism in Saccharomyces cerevisioe. Proc. Natl. Acad. Sci. U.S.A. 104, 2402-2407

21 Heinrich, R. and Klipp, E. (1996) Control analysis of unbranched enzymatic chains in states of maximal activity. J. Theor. Biol. 182 243-252

22 Dedeken, R.H. (1966) effect: a regulatory system in yeast. J. Gen. Microbiol., Crabtree 44, 149-156

23 Jensen, P.R., Westerhoff, H.V. and Michelsen, O. (1993) Excess capacity of $\mathrm{H}^{+}$-ATPase and inverse respiratory control in Escherichio coli. EMBO ) 12, $1277-1282$

24 Molenaar, D., van Berlo, R., de Ridder, D. and Teusink, B. (2009) Shifts in growth strategies reflect tradeoffs in cellular economics. Mol. Syst. Biol. 5, 323

25 Snoep, J.L., Yomano, L.P., Westerhoff, H.V. and Ingram, L.O. (1995) Protein burden in Zymomonas mobilis: negative flux and growth control due to overproduction of glycolytic enzymes. Microbiology 141, 2329-2337

26 Westerhoff, H.V., Winder, C., Messiha, H., Simeonidis, E., Adamczyk, M., Verma, M., Bruggeman, F.J. and Dunn, W. (2009) Systems biology: the elements and principles of Life. FEBS Lett. 583, 3882-3890

27 Kell, D.B. and Oliver, S.G. (2004) Here is the evidence, now what is the hypothesis? The complementary roles of inductive and hypothesis-driven science in the post-genomic era. BioEssays 26, 99-105

Received 8 March 2010

doi: $10.1042 /$ BST0381225 